\title{
What is novel in the novelty oddball paradigm? Functional significance of the novelty P3 event-related potential as revealed by independent component analysis
}

\author{
Stefan Debener ${ }^{\mathrm{a}, \mathrm{b}, *}$, Scott Makeig ${ }^{\mathrm{c}}$, Arnaud Delorme ${ }^{\mathrm{c}}$, Andreas K. Engel ${ }^{\mathrm{a}, \mathrm{b}}$ \\ a Institute of Neurophysiology and Pathophysiology, Center of Experimental Medicine, University Hospital Hamburg-Eppendorf, D-20246 Hamburg, Germany \\ ${ }^{\mathrm{b}}$ Institute of Medicine, Research Center Juelich, D-52428 Juelich, Germany \\ ${ }^{\mathrm{c} S}$ Swartz Center for Computational Neuroscience, Institute for Neural Computation, University of California San Diego 0961, La Jolla, CA 92093-0961, USA
}

Accepted 7 September 2004

Available online 13 October 2004

\begin{abstract}
To better understand whether voluntary attention affects how the brain processes novel events, variants of the auditory novelty oddball paradigm were presented to two different groups of human volunteers. One group of subjects $(n=16)$ silently counted rarely presented 'infrequent' tones $(p=0.10)$, interspersed with 'novel' task-irrelevant unique environmental sounds $(p=0.10)$ and frequently presented 'standard' tones $(p=0.80)$. A second group of subjects $(n=17)$ silently counted the 'novel' environmental sounds, the 'infrequent' tones now serving as the task-irrelevant deviant events. Analysis of event-related potentials (ERPs) recorded from 63 scalp channels suggested a spatiotemporal overlap of fronto-central novelty P3 and centro-parietal P3 (P3b) ERP features in both groups. Application of independent component analysis (ICA) to concatenated single trials revealed two independent component clusters that accounted for portions of the novelty P3 and P3b response features, respectively. The P3b-related ICA cluster contributed to the novelty P3 amplitude response to novel environmental sounds. In contrast to the scalp ERPs, the amplitude of the novelty P3 related cluster was not affected by voluntary attention, that is, by the target/nontarget distinction. This result demonstrates the usefulness of ICA for disentangling spatiotemporally overlapping ERP processes and provides evidence that task irrelevance is not a necessary feature of novelty processing.
\end{abstract}

(C) 2004 Elsevier B.V. All rights reserved.

Keywords: ICA; ERP; Single-trial analysis; P300; Novelty P3; P3b; Spatiotemporal overlap

\section{Introduction}

As yet, little is known about how the brain processes novelty (e.g., Ref. [36]). Unexpected novel events elicit a cascade of reactions known as the orienting response,

Abbreviations: EEG, electroencephalogram; EEGLAB, open source EEG analysis toolbox (http://www.sccn.ucsd.edu); ERP, event-related potential; FP3, frontal P3 independent component cluster; ICA, independent component analysis; PCA, principal component analysis; PP3, parietal P3 independent component cluster; PVAF, percentage of variance accounted for

* Corresponding author. Institute of Neurophysiology, University Hospital Hamburg-Eppendorf, Martinistr. 52, D-20246 Hamburg, Germany. Fax: +49 40428037722

E-mail address: s.debener@uke.uni-hamburg.de (S. Debener). which serve to prepare the organism for sudden changes in the environment [38]. The novelty P3 event-related potential (ERP) can be regarded as the brain-electrical correlate of the orienting response [18]. In this paradigm, the subject's voluntary attention is directed to a rarely presented 'target' stimulus, while his/her brain-electrical response to unexpectedly occurring 'novel' stimuli is investigated. Novel stimuli are usually more complex than target and standard stimuli, and they are made unique by being never repeated. In contrast to targets, novel stimuli are irrelevant to the subject's task. Thus, the novel stimuli are characterized by low probability, task irrelevance and contextual salience, whereas the target stimulus occurs with equally low probability, but is task-relevant and familiar. 
As first described by Courchesne et al. [5], taskirrelevant unique novel stimuli elicit a positive ERP deflection with a fronto-central maximum and a latency of about 250 to $400 \mathrm{~ms}$. This novelty P3 ERP deflection has been distinguished from the $\mathrm{P} 3 \mathrm{~b}$, which has a centralposterior maximum between 300 and $600 \mathrm{~ms}$ and is commonly evoked by target stimuli (see Ref. [18] for review). The novelty P3 is reduced following repeated stimulus presentations [24] and becomes smaller in the second as compared to the first half of an experimental session [6], reflecting habituation of the orienting response. Hence, the novelty P3 seems related to an involuntary, stimulus-driven, or bottom-up attention-orienting mechanism. In comparison, P3b amplitude does not habituate within experimental sessions [6], and is thought to be evoked whenever events force an updating of stimulus representations held in working memory [12].

Regarding the functional significance of the novelty P3, two models can be distinguished [20]. In the attention switching model (ASM), it is proposed that the novelty P3 reflects involuntary switching of attention to deviant events. Task-irrelevant deviant events that distract the subject from a primary task elicit a novelty P3 [13]. Moreover, complex novel stimuli have been found to result in a larger novelty P3 as compared to simple tonal stimuli, which has been interpreted as reflecting increased task distraction. Consequently, the amplitude of the novelty P3 is thought to reflect the amount of involuntary attention switching [15]. This model, however, cannot explain why the novelty P3 is larger in passive attention as compared to ignore novelty oddball experiments (e.g., Refs. [21,35]). For instance, Holdstock and Rugg [21] compared the novelty P3 during passive listening to an auditory novelty oddball series (passive attention) with the novelty P3 while subjects were engaged in a computer game (ignore condition). The novelty $\mathrm{P} 3$ amplitude in response to rarely presented novel stimuli was larger when subjects passively attended the stimuli than when the stimuli were ignored, a finding incompatible with the ASM. To account for such results, an alternative, the response-inhibition model (RIM), has been proposed [20]. In this model, it is assumed that the detection of a deviant event leads automatically to context updating, which is assumed to be reflected by the P3b. Thorough processing and proper identification of the novel stimulus then reveals that a response should not be executed. Consequently, a (response) mechanism activated by deviance detection needs to be inhibited. According to this view, the novelty $\mathrm{P} 3$ is a manifestation of this inhibitory process [20]. Note also that the RIM assumes that both novelty P3 and P3b ERP components are elicited by task-irrelevant novel stimuli.

From both models, it can be predicted that task irrelevance of salient stimuli is necessary to elicit a novelty P3. The more irrelevant salient stimuli are, the larger the novelty P3 response should be. Thus, stimuli eliciting a novelty P3 should not be relevant for the task performed, since distraction from a primary task is the core feature of the ASM, and inhibition of an early initiated response is the main feature of the RIM.

Recent studies applying principal components analysis (PCA) of ERPs revealed that deviant stimuli elicit both novelty P3 and P3b ERP features. This in turn suggests that the scalp-recorded ERP reflects both processes for both target and nontarget novel conditions [20,39,40]. In addition, the investigation of task relevance on the novelty P3 is hampered by the fact that this deflection partly overlaps with the P3b in time and space [12]. Because taskrelevant stimuli evoke a $\mathrm{P} 3 \mathrm{~b}$, it is conceivable that, on the scalp level, task-relevant novel stimuli evoke an artificially "larger" novelty P3, due to the spatiotemporal overlapping P3b. Thus, inferences on the functional significance of the novelty $\mathrm{P} 3$ based on scalp voltage data alone can be misleading.

In the present study, we investigated whether the novelty P3 is modulated by task relevance. Specifically, data from an auditory novelty oddball paradigm obtained from two separate subject groups were analyzed, in order to compare responses to the same novel stimuli for target and nontarget conditions. For one group, infrequent presentations of unique environmental sounds were designated as 'target' stimuli, and an infrequently presented sinusoidal tone served as rare 'nontarget'. For the other group, the rare tones were designated as targets and the unique environmental sounds served as nontarget stimuli. Using independent component analysis (ICA), a recently developed electroencephalogram (EEG) signal analysis procedure [8,28,31,32], we investigated whether a spatiotemporally overlapping P3b feature could account for the predicted larger amplitude of the novelty P3 feature in response to target as compared to nontarget novel stimuli.

\section{Methods}

\subsection{Subjects}

Healthy volunteers were recruited from the Research Center Juelich and from a local college. Screening confirmed that subjects were free of past or current psychiatric and neurological disorders. All participants had normal hearing. Data were recorded from 39 individuals. Because of technical problems or excessive artifacts, six data sets were excluded from further analysis. Participants were randomly assigned to one of two groups. Group 1 consisted of 16 participants ( 8 female, 8 male) with a mean age of 24.1 years (range: $18-34$ years). Group 2 consisted of 10 female and 7 male participants with a mean age of 22.8 years (range: 19-28 years). Written informed consent was obtained from all participants prior to the start of the experiment. The study was performed in agreement with local ethical standards and the 1964 Declaration of Helsinki. Volunteers were paid 16 EUR for their participation. 


\subsection{Stimuli and task}

All subjects performed an auditory novelty oddball task. Stimuli were presented binaurally at about $70 \mathrm{~dB}$ through foam-protected air-tube insert earphones. Two sinusoids of $339 \mathrm{~ms}$ duration $(350$ and $650 \mathrm{~Hz})$ served as frequent and rare tones, respectively. Assignment of the two tone frequencies to the rare or frequent stimulus class was counterbalanced across subjects. Probability of rare tones was $10 \%$ and probability of occurrence of frequent tones was $80 \%$. The third class of stimuli (probability $10 \%$ ) consisted of 90 unique, novel environmental sounds (mean duration: $338 \mathrm{~ms}$, range: $161-402 \mathrm{~ms}$ ), provided to the authors by Fabiani et al. [16]. Each environmental sound was presented once in pseudo-randomized order, and belonged to six different categories (animal, machine, music, human, electronic, bird call). The experimental session was subdivided into five task blocks consisting of 180 stimuli each, separated by 1-min breaks. Interstimulus interval varied pseudo-randomly between 960 and $1360 \mathrm{~ms}$ in 100 -ms steps.

The subjects' task was to silently count the rare tones (Group 1), or the rare novel environmental sounds (Group 2). A between subjects design was chosen to prevent effects of task order, to keep the recording duration short, and to analyze the effects of task relevance on the same set of novel stimuli without within-subject repetition. Accordingly, for Group 1 the rare tones were task-relevant and familiar, and the environmental sounds were taskirrelevant and novel, as in previous novelty oddball studies [6]. For Group 2, target and nontarget assignment was reversed. The novel environmental sound stimuli were defined as targets and the rare tones were taskirrelevant. To ensure that tones and sounds were correctly identified by the subjects, three test stimuli of each category, that is, standards, rare tones and novel sounds, were presented with a verbal description of each stimulus category on the computer screen prior to the first block. To maintain attention to the targets across blocks, between 0 and 3 additional target stimuli were included in each block. These (dummy) trials were excluded from EEG analysis. After each block, the subjects were asked how many targets they had detected and their answers were recorded.

\subsection{EEG recording}

EEG was recorded from 63 equidistant scalp sites (spacing, $37 \pm 3 \mathrm{~mm}$ ) using high input impedance amplifiers (200 M , Electrical Geodesics, Eugene, OR, USA) and Ag/ $\mathrm{AgCl}$-electrodes mounted in an electrode cap (FMS, Munich, Germany). Eye blinks were monitored using an additional electrode placed below the right eye. The nose-tip served as reference and ground was placed at the left mastoid. All electrode impedances were reduced to $20 \mathrm{k} \Omega$ before data acquisition [17]. Data were recorded with a bandpass of 0.1 to $100 \mathrm{~Hz}$ and digitized at $500 \mathrm{~Hz}(0.024$ $\mu \mathrm{V}$ precision) for later off-line analysis.

\subsection{Data analysis}

EEG data analysis was performed using EEGLAB 4.311, a freely available open source toolbox (http:// www.sccn.ucsd.edu/eeglab) running under Matlab 6.3 (The Mathworks). A detailed outline of the major concepts implemented in EEGLAB is provided by Delorme and Makeig [8]. First, data were down-sampled to $250 \mathrm{~Hz}$ to save later computation time, and were then digitally filtered using a bidirectional linear filter that preserves the phase information (pass band 0.3 to $40 \mathrm{~Hz}$ ) to minimize drifts and line noise, both being present in few data channels. Data epochs were extracted ( -200 to 800 $\mathrm{ms}$ ) and baseline corrected ( -200 to $0 \mathrm{~ms}$ ). To achieve a comparable signal-to-noise ratio across experimental conditions, the total number of standard trials (720) was reduced to those 90 trials preceding the rare tones, to equalize the number of stimuli for each condition. The remaining total of 270 epochs in each individual data set was then visually inspected. Epochs containing unique, non-stereotyped artifacts (e.g., swallowing, electrode cable movements, etc.) were rejected from further analysis, whereas epochs containing repeatedly occurring, stereotyped artifacts (e.g., eye blinks, heart beat artifacts, etc.) were corrected using ICA $[8,22]$. Following this procedure, the mean percentage of rejected epochs was $7.4 \%$ (mean: 19.97, range: 2-62). A few bad channels were also excluded from further analysis $(2.22 \%$; range: $0-7)$.

Next, individual concatenated single-trial data sets were decomposed using infomax ICA. Infomax ICA exploits temporal independence to perform blind source separation $[4,28,29]$. It finds a square unmixing matrix that maximizes the joint entropy of zero-mean input vectors, and can decompose linearly mixed processes having non-gaussian distributions. The fitness of this approach for the analysis of EEG data has been demonstrated repeatedly (e.g., Refs. $[7,22,23,30,31])$. ICA decomposition of EEG data provides spatially fixed and temporally independent components without a priori assumptions on the temporal dynamics or spatial structure of the underlying processes. For the present data, extended ICA was performed on individual data sets varying in size from 56 to 64 channels and from 202 to 268 1 -s data epochs ( -200 to $800 \mathrm{~ms}$ ). The initial learning rate was gradually reduced to a stopping weight change below $10^{-7}$ over 150 or more training iterations. These settings, performed using the EEGLAB function runica.m [8,29], were found to produce robust decompositions. For further details of the ICA artifact correction approach employed here, see Ref. [22].

For each individual data set, ICA derives as many components as there are channels in the training data. Thus, in the present data, a total of 2065 components were derived from the 33 subjects' data. A semiautomatic multilevel 
approach, applied separately to each group, was then performed to define clusters of relevant independent components. First, components representing artifacts were identified and rejected from further analysis by visual inspection of individual component properties: the combined analysis of a component map (the inverse weight matrix for the component), the corresponding component ERP (time-domain average of the activity time course), the component power spectrum (frequency-domain average of the component activity) and the component ERP-image, the latter being a method for visualizing event-related signal variations across single trials [23,30]. Artifacts typically produce prominent signs in one or more of these data representations and can therefore be easily detected. This analysis revealed a total of 840 artifactual components (mean of 25 per subject), which were rejected from further analysis. A mean of 37 independent components (range: 27-50) for each individual data set remained. For the calculation of scalp ERPs, artifact-free EEG data were obtained by back-projecting the remaining non-artifactual ICA components. This was done by multiplying the selected component activities with the reduced component mixing matrix.

Ideally, an independent component should account for synchronous activity within a connected cortical domain, and accordingly its scalp projection should match a single equivalent current dipole (or sometimes two bilaterally symmetric dipoles). In practice, many biologically plausible independent components are characterized by scalp maps fitting the projection of a single equivalent current dipole [8,31]. Therefore, the goodness of fit for modeling each independent component scalp map with a single equivalent current dipole was used to quantify component quality. This was done by submitting individual component maps to an automatic single dipole source localization algorithm (DIPFIT, contributed by R. Oostenveld et al.) as implemented in EEGLAB, using a standard four-shell spherical head model as implemented in commercial software packages (radii in mm: 71, 72, 79, 85; conductivity in S/m: 0.33, $1,0.0042,0.33)$. From 1225 component maps modeled with single equivalent current dipoles, a total of 341 components fitted with a residual variance of less than $10 \%$ (mean residual variance: $5.48 \%$; range: $0.9-9.9$ ). Only these components were considered further, reducing the mean number of remaining components for each subject to approximately 10 (range: 5-15).

Seven distinct component clusters were then defined by combined inspection of component dipole location and orientation, scalp map, ERP time course and spectrum. These clusters were defined by their most prominent activity features, as left-occipital alpha, central-occipital alpha, right-occipital alpha, left mu, right $\mathrm{mu}$, frontal P3 and parietal P3. The robustness of this clustering result was investigated by repeating the above-described procedure for the data from subject Group 2 and comparing the results. Several of the clusters identified strongly resembled clusters that have been described earlier in analyses of data collected in a different task with a sparser electrode array $[31,33]$. Application of a fully automatic clustering algorithm (based on the Mahalanobis distance measure) partly reproduced the above-described cluster results, but was found to be less satisfactory. The present report considers the two ICA clusters, which explained most ERP variance, as reflecting the main features of P3b and novelty P3.

The percentage of variance accounted for (PVAF) in the ERP data by a single independent component was calculated as follows: PVAF $=100 \times(1-\operatorname{var}($ Data - comp $) /$ $\operatorname{var}($ Data)). "Comp" refers to a two-dimensional array (channels $\times$ data points) depicting the back-projected activity contributed to the ERP by the component; "var" is the variance; and "Data" is a two-dimensional array (channels $\times$ data points) referring to the artifact-corrected singletrial data at all channels (from 0 to $800 \mathrm{~ms}$ post stimulus onset). To compute the PVAF of two or more components, the same formula was applied to the summed backprojections of the components of interest. Note that the PVAF of two or more components is not equal to the sum of PVAF of these components.

\section{Results}

Behavioral analysis revealed that the mean target count was between $96 \%$ and $98 \%$ of the correct number, indicating low task difficulty as expected from an earlier study [6]. Between 22 and 27 out of 33 subjects did not make any errors, that is, target count was $100 \%$ for the majority of the subjects in most blocks. Because of the resulting lack of variance in the behavioral data, no further statistical analyses of task performance were conducted.

\subsection{Scalp ERPS}

As lateralized ERPs were neither expected [19] nor seen, Fig. 1 shows the ERPs for frequent tones, rare tones and rare sounds at selected midline scalp sites. The two groups did not significantly differ with regard to the relatively early auditory ERP features P1, N1 and P2, neither in the frequent standard tone condition (left column) nor in the rare tone (middle column) or novel sounds condition (right column). As compared to the standard tones, a more complex ERP pattern emerged in response to the rare tones, which served as targets in Group 1 and as nontargets in Group 2. A frontocentral N2 response was followed by a central positivity peaking at E34 $(\mathrm{Cz}, 336 \mathrm{~ms}$ latency and $7.81 \mu \mathrm{V}$ amplitude in Group 1; $320 \mathrm{~ms}, 6.98 \mu \mathrm{V}$ in Group 2), which we classified as P3a [41]. Shortly following the P3a ERP deflection, differences between groups became significant, as indicated by plotted horizontal grey bars showing pointwise $T$-test results with significance level $p<0.01$. Group 1 showed a significantly larger parietal positivity following rare tones than Group 2 for whom the rare tones were 
Frequent Tones

Rare Tones

- Group $1(n=16)$; Count rare tones

- Group $2(n=17)$; Count novel sounds
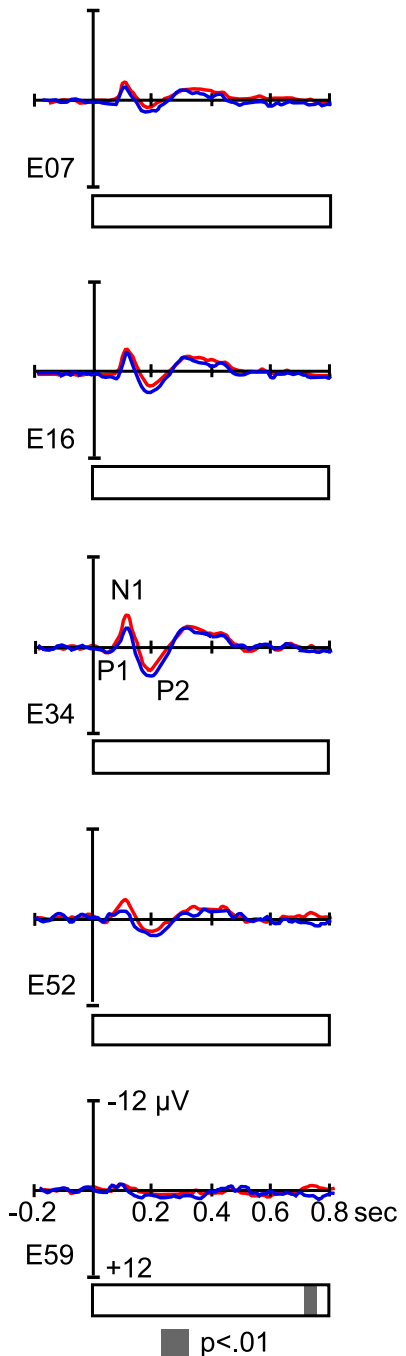
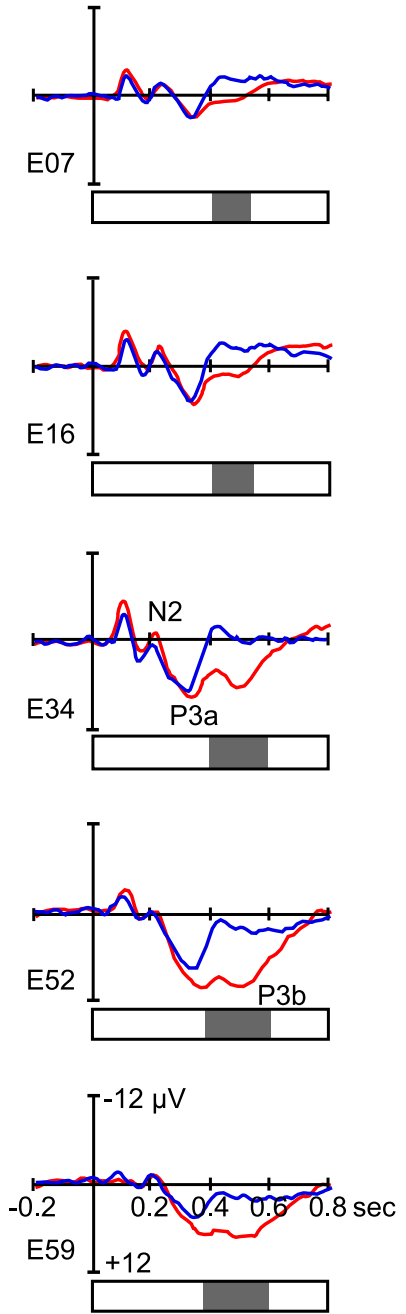

Novel Sounds
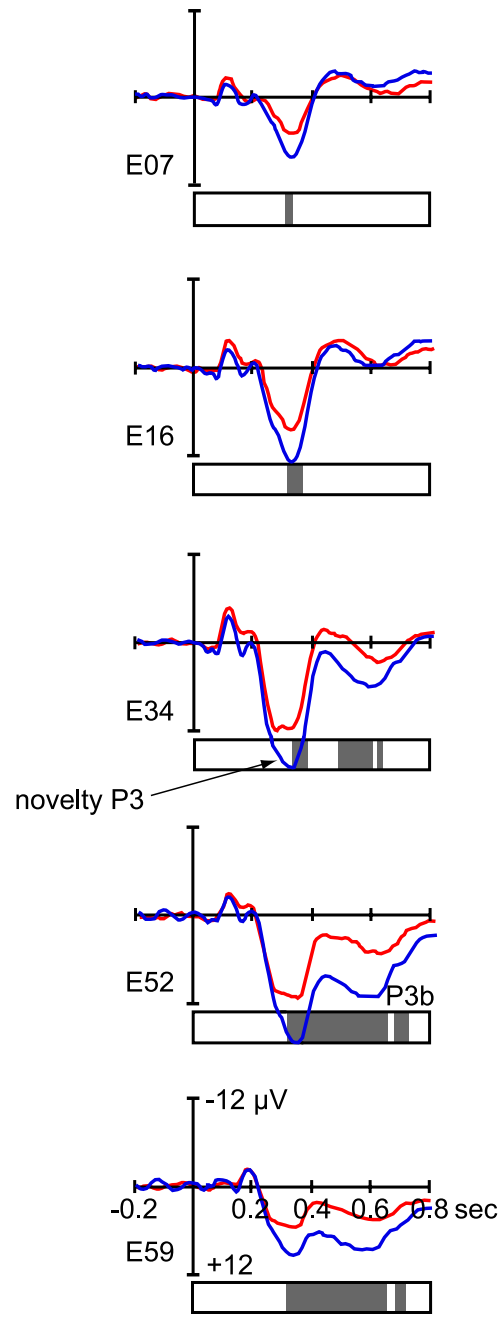

Fig. 1. Grand mean ERPs at midline scalp sites for three experimental conditions: responses to frequent tones (left column), rare tones (middle), and rare unique 'novel' sounds (right). Red lines show grand mean ERPs for Group $1(n=16)$, for whom rare tones were the targets of manual responses. Superimposed blue lines give the grand mean ERPs for Group $2(n=17)$, for whom rare novel sounds were the target stimuli. Electrode E34 is equivalent to Cz, and electrode locations E07 and E59 are close to locations Fz and Oz of the International 10-20 system, respectively. Horizontal bars below each trace represent $t$-test results from 0 to $800 \mathrm{~ms}$ post stimulus onset, with values $p<0.01$ represented in grey, to illustrate the time course of significant group differences.

nontargets. This difference was most prominent at electrode E52 (close to $\mathrm{Pz}$ of the International 10-20 system). This parietal ERP deflection was considered a P3b [41], and peaked at $360 \mathrm{~ms}(10.95 \mu \mathrm{V})$. For Group 2, a positivity resembling the $\mathrm{P} 3 \mathrm{~b}$ could not be identified unambiguously.

Analysis of the grand mean ERPs in response to the unique environmental sounds (Fig. 1; right column) revealed a large central positive deflection in both groups, further referred to as novelty P3 [5]. For Group 1 at E34, the novelty P3 ERP had a double-peak morphology, with a maximum at $268 \mathrm{~ms}(12.25 \mu \mathrm{V})$ closely followed by a second peak at $308 \mathrm{~ms}(12.04 \mu \mathrm{V})$. The second peak was also clearly evident in the ERPs of Group 2, for whom the environmental sounds served as targets. The novelty P3 peak (17.69 $\mu \mathrm{V}$ at $320 \mathrm{~ms})$ was significantly larger at all midline scalp sites for Group 2 as compared to Group 1. However, a significant difference between both groups was not only evident for the novelty P3, but also for the P3b at central and parietal scalp sites. As expected, a larger P3b was found for Group 2 (E52: $11.48 \mu \mathrm{V}$ at $600 \mathrm{~ms}$ ), which had the novel environmental sounds as targets. Taken together, the component morphology depicted in Fig. 1, along with the significance pattern of group differences, supports the assumption that spatiotemporal overlap of the novelty $\mathrm{P} 3$ and $\mathrm{P} 3 \mathrm{~b}$ response peaks occurred, in particular in the Group 2 responses to novel sounds. 


\subsection{Analysis of independent component clusters}

From the seven component clusters identified, five closely resembled those reported previously (left occipital alpha, central occipital alpha, right occipital alpha, left central mu, right central mu $[31,33])$. However, as the goal of this report was to describe and investigate the main ICA features related to novelty P3 and P3b ERP components, these other clusters were not considered further. Two clusters showed obvious similarities to the scalp ERPs novelty P3 and P3b, and were labeled frontal P3 (FP3) and parietal P3 (PP3) cluster. Fig. 2A details the average characteristics of the FP3 cluster as identified in Group 1, across all three experimental conditions. The FP3 cluster consisted of 21 independent components from 15 out of 16 subjects. The normalized grand-mean component ERP (Fig. 2A, upper left inset) revealed an early negative deflection at $100 \mathrm{~ms}$, followed by a large positive deflection between 200 and 400 ms post stimulus onset, peaking at $260 \mathrm{~ms}$. Note the similarity of the FP3 shape and the scalp novelty P3 (Fig. 1). The FP3 cluster had a mid fronto-central topography (Fig. 2A, top middle inset) typical of a novelty P3 ERP, and a smooth mean spectrum with a small $10-\mathrm{Hz}$ peak (Fig. 2A, top right inset). Individual equivalent dipole locations of the components forming the FP3 cluster, plotted on a meanMRI brain image (Montreal Neurological Institute, Canada), clustered along the fronto-central midline, close to the anterior cingulate and pre-supplementary motor area. However, note the variation in dipole locations, as illustrated in the coronal view (Fig. 2A, bottom row). In summary, the FP3 cluster characteristics outlined in Fig. 2A strongly suggested a relation to the scalp novelty P3.

Fig. 2B shows the second cluster of interest, labeled parietal P3 (PP3) due to its time course and topography. Twenty-four components from 14 subjects contributed to the PP3 cluster, which was characterized by a later and slower ERP positivity. Note the early onset and long latency of the PP3 component ERP, starting at about 200 $\mathrm{ms}$ and lasting for $600 \mathrm{~ms}$ before returning to baseline, with a peak latency at $456 \mathrm{~ms}$ (Fig. 2B, upper left inset). Furthermore, a bilateral parietal topography (middle inset) and a mean spectrum with a small $10-\mathrm{Hz}$ peak (right inset)
A. Frontal P3 cluster (FP3): Group 1 (count rare tones)
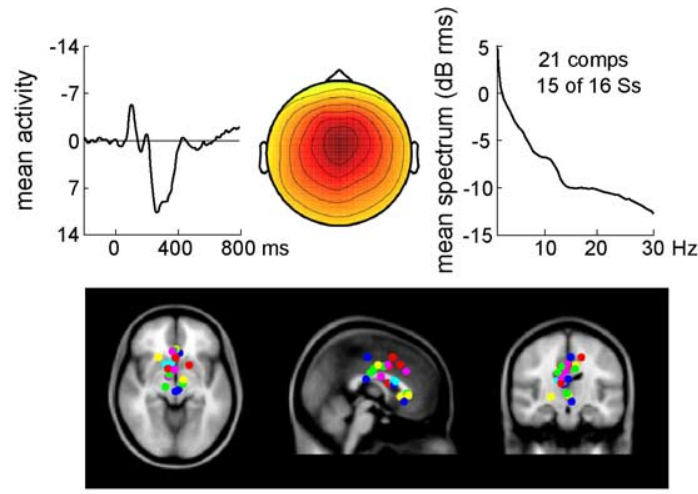

B. Parietal P3 cluster (PP3)
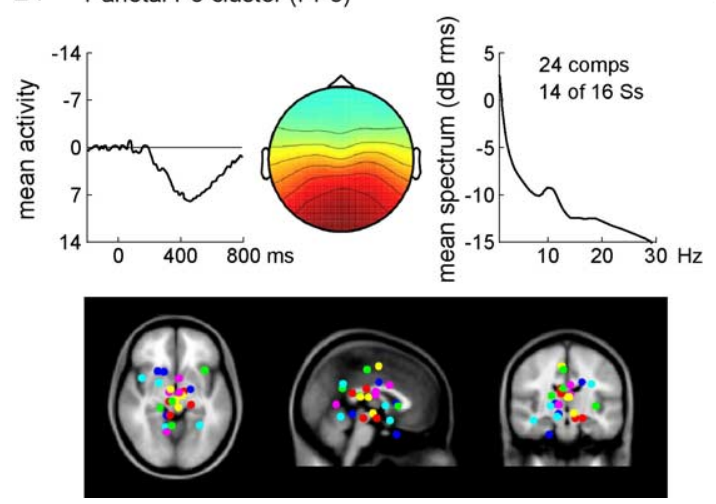

C. Frontal P3 cluster (FP3): Group 2 (count novel sounds)
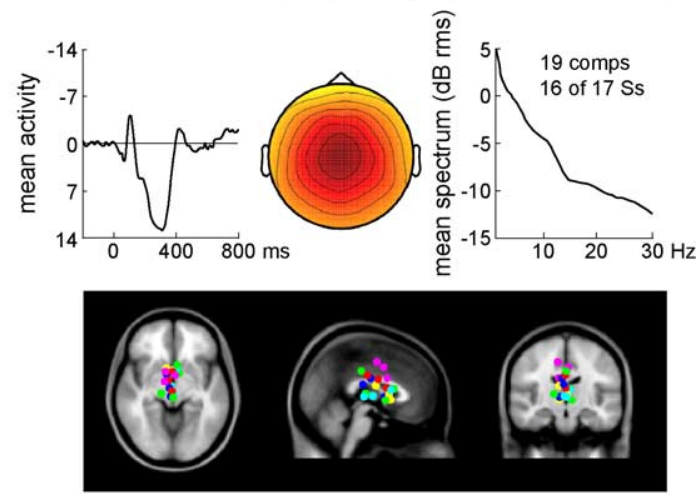

D. Parietal P3 cluster (PP3)
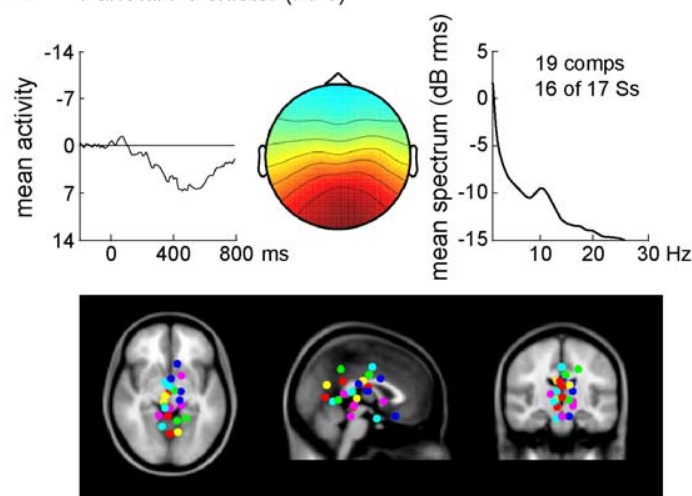

Fig. 2. Independent component clusters, frontal P3 (FP3, A, C) and parietal P3 (PP3, B, D) identified from Group 1 (A, B) and Group 2 (C, D). Upper row of the panel for each group shows the grand mean component cluster ERP (upper left), scalp map (middle), and activity spectrum (right). The lower row shows single equivalent current dipole locations for each component contributing to the corresponding cluster, plotted on a mean MRI image (Montreal Neurological Institute) from three different perspectives (top, sagittal and coronal views). Individual trials were normalized to unit variance in the prestimulus baseline, for illustration of average cluster ERP activity, by dividing component ERPs by the standard deviation of the prestimulus baseline ( -200 to $0 \mathrm{~ms}$ ), and for illustration of average cluster topography by dividing each map by its RMS. For the grand mean spectra, the amplitude of each spectrum was scaled by the corresponding component RMS scalp power. 
characterized this cluster. The dipole locations of the independent components forming the PP3 cluster for Group 1 are depicted at the bottom row of Fig. 2B. As compared to the FP3 cluster, PP3 cluster dipole locations were more dispersed across different brain regions, though centered in the posterior midline. Its time course, which strongly resembled a prototypic $\mathrm{P} 3 \mathrm{~b}$ time course, and parietal topography suggested that the PP3 cluster contributed to the scalp P3b ERP deflection.

The ICA decomposition of the Group 2 data revealed similar FP3 and PP3 component clusters as for Group 1. Here, the FP3 cluster consisted of 19 components from all 16 subjects. The PP3 cluster consisted of 23 components from 15 out of 16 subjects. Between-group differences in mean component cluster topographies are barely visible, suggesting a good reproducibility of both component cluster characteristics. For the FP3 cluster of Group 2 (Fig. 2C), a negative peak at $104 \mathrm{~ms}$ was again followed by a large and sharp positive deflection between 200 and $400 \mathrm{~ms}$ post stimulus onset (peak latency $308 \mathrm{~ms}$ ), resembling a novelty P3 ERP. The mean spectrum of the FP3 cluster was again smooth, and most of the individual dipoles were again located close to the fronto-central midline. A similarly striking congruence was evident for the PP3 clusters. For Group 2 (Fig. 2D), a late positivity again started relatively early (about $200 \mathrm{~ms}$ ), peaked at 484 $\mathrm{ms}$ and lasted roughly $600 \mathrm{~ms}$. Thus, the two clusters FP3 and PP3 were reproduced in two independent samples, and resembled the well-known scalp ERP features novelty P3 and $\mathrm{P} 3 \mathrm{~b}$, respectively.

\subsection{Contribution of independent components to experimental conditions}

The contributions of each cluster to ERP responses for both rare event classes were then investigated, again separately for both groups (Fig. 3). For Group 1, the FP3 cluster, back-projected to the scalp, explained $30 \%$ of the scalp ERP variance in the 0 to $800 \mathrm{~ms}$ latency range for the nontarget novel sounds condition, and $18 \%$ in the target rare tones condition. The PP3 cluster, on the other hand, explained more variance in the target rare tones condition (i.e., 19\%) as compared to the nontarget novel sounds condition (i.e., 10\%). A somewhat different pattern of results emerged for Group 2. Here, the FP3 cluster explained $31 \%$ of the ERP variance in the target novel sounds condition, and $23 \%$ in the nontarget rare tones condition. Note that the latter condition comprised a P3a scalp ERP feature (cf. Fig. 1). As can also be seen in Fig. 3 , the PP3 cluster explained $12 \%$ in the target novel sounds condition, and $10 \%$ in the nontarget rare tones condition.

For Group 1, joint back-projection of both clusters explained $48 \%$ of the ERP variance in the target rare tones condition, and $51 \%$ of the variance in the nontarget novel sounds condition. The PP3 cluster contributed more to the target than the nontarget condition. The FP3 cluster, which also explained a considerable amount of variance in both conditions, contributed more to the nontarget condition. Summing the grand mean back-projections of the FP3 and PP3 clusters for Group 2 revealed that $32 \%$ of the ERP variance in the nontarget rare tones condition and $48 \%$ in the target novel sounds condition was accounted for by the two component clusters. Taken together, this pattern confirmed that the FP3 cluster was primarily related to the novelty P3 ERP, whereas the PP3 cluster reflected major aspects of the P3b ERP deflection.

\subsection{Statistical analyses}

To study the statistical significance of these effects, the contribution of the back-projected FP3 and PP3 components to the grand mean ERPs was computed by calculating the mean across the maximum ERP envelope in the time intervals $230-360 \mathrm{~ms}$ for FP3, and 400-580 ms for PP3. These intervals were used previously to quantify scalp ERP novelty P3 and P3b components, respectively [6]. For Group 1, a consecutive two-way ANOVA using the repeated measurements factors Cluster (FP3, PP3) and Condition (sounds, tones) revealed, aside from significant main effects for Cluster, $F_{1,20}=8.86, p=0.007, \eta^{2}=0.31$, and Condition, $F_{1,20}=12.74, p=0.002, \eta^{2}=0.39$, a significant interaction of Cluster and Condition, $F_{1,20}=37.43$, $p<0.001, \eta^{2}=0.65$. This analysis statistically confirmed that FP3 contributed more to the novel sounds response, whereas PP3 contributed more to the rare tones response. The two-way ANOVA Cluster $\times$ Condition for Group 2 paralleled the findings for Group 1. A significant main effect for Cluster, $F_{1,18}=13.75, p=0.002, \eta^{2}=0.43$, and Condition, $F_{1,18}=26.61, p<0.001, \eta^{2}=0.60$, emerged. Again, the interaction Cluster $\times$ Condition was significant, $F_{1,18}=45.05, p<0.002, \eta^{2}=0.42$. An additional analysis was performed to investigate whether the PP3 cluster reflected the enhanced novelty P3 ERP of Group 2 as compared to Group 1, by comparing the back-projection of the FP3 cluster with the joint back-projection of both FP3 and PP3 cluster in the rare sounds condition. A Group $\times$ Cluster ANOVA, performed for the maximum ERP envelope in the mean time interval $230-360 \mathrm{~ms}$, revealed a significant Cluster main effect, $F_{1,31}=18.69, p<0.001, \eta^{2}=0.38$, indicating higher amplitudes for the joint back projection of FP3 and PP3 clusters as compared to the back projection of the FP3 cluster. Neither the main effect Group nor the interaction Cluster $\times$ Group approached significance (both $F_{\mathbf{S}}<1$ ).

A main question of this study was whether the brain processes reflected by the novelty P3 ERP are altered by voluntary attention. Accordingly, the responses of both groups to the rare novel sounds, as expressed in the FP3 cluster, were compared. As shown in Fig. 3, this comparison revealed that the FP3 cluster in Group 1 and Group 2 explained almost the same amount of 
A.

Group 1 (count rare tones)
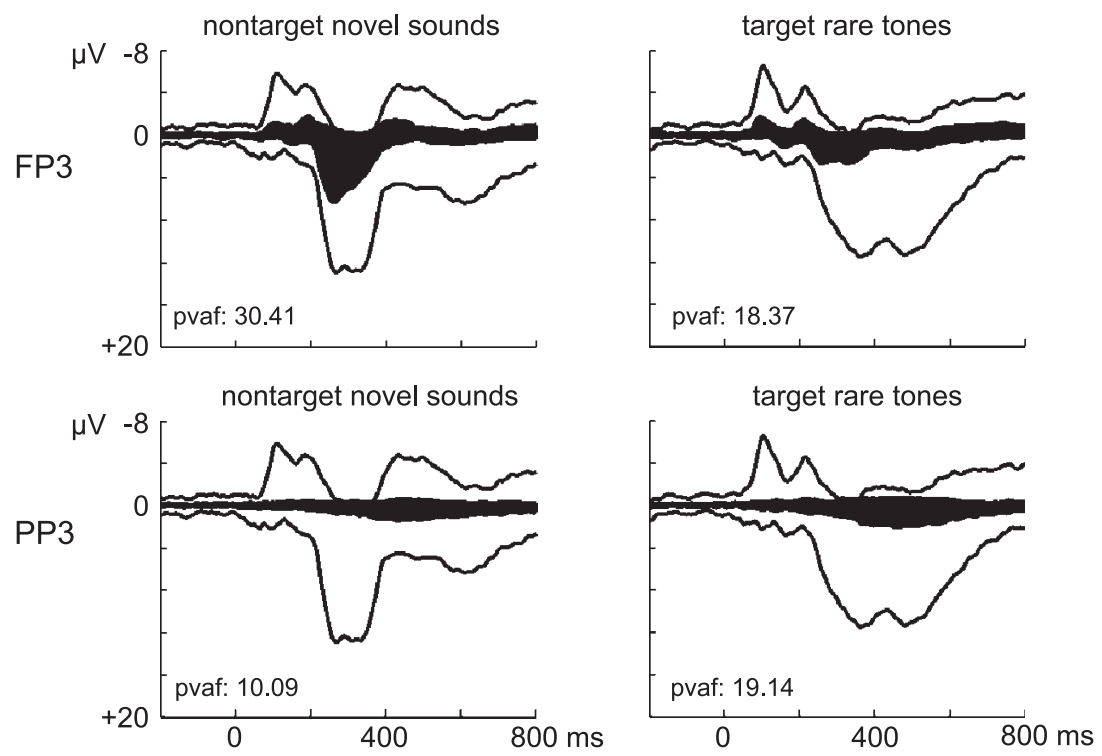

B. Group 2 (count novel sounds)
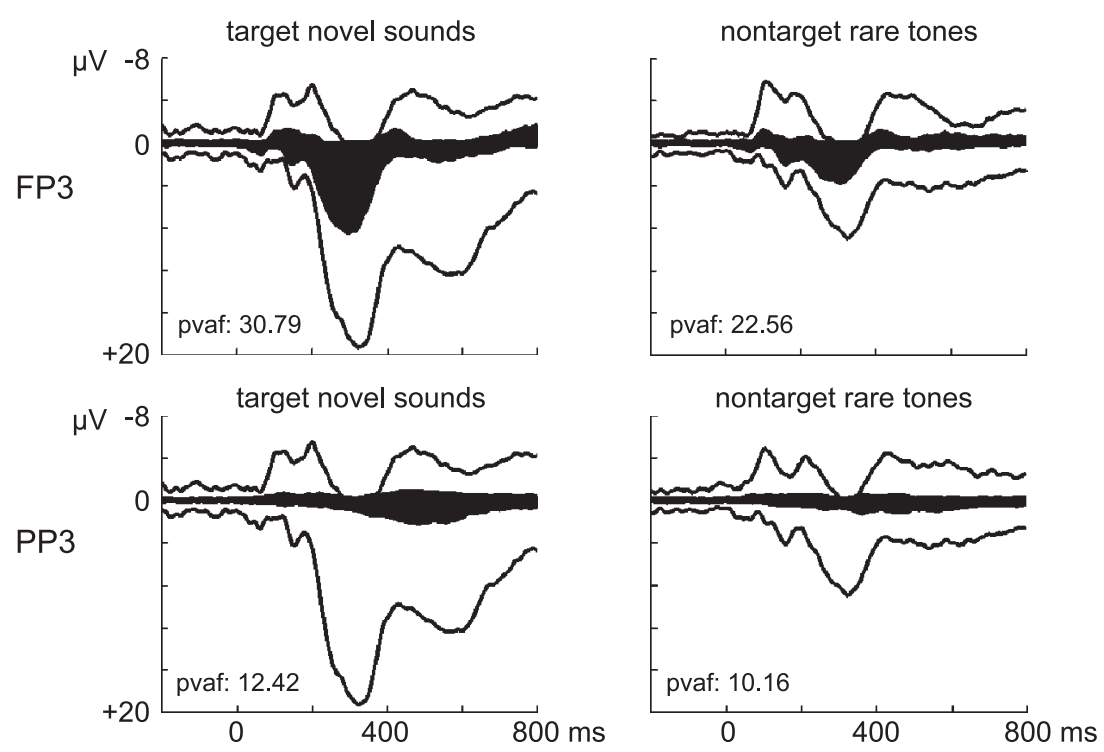

Fig. 3. (A, B) Grand mean FP3 and PP3 cluster ERP envelopes plotted separately for both stimulus conditions and both subject groups. The two outer traces in each plot represent the scalp-recorded data envelope, that is, the minimum and maximum potentials at all channels at each latency. The top and bottom of the black-filled areas give the envelope of the grand mean activity of the back-projected independent components in the corresponding cluster. The mean 'percent variance accounted for' (PVAF) by the independent components is given.

variance $(30.41 \%$ versus $30.79 \%)$. The difference in the novel sounds condition between the back-projected FP3cluster ERPs of both groups was not statistically significant, $t_{38}=0.27$, ns.

\subsection{Habituation}

To analyze the temporal dynamics of the FP3 cluster activity, in particular its variation with time-on-task, ERPimage plots $[23,30]$ were created of the activity time courses of these components in single trials. As can be seen in the upper part of Fig. 4, for Group 1, FP3 showed a constant stimulus-onset related activity, giving rise to the relatively sharp positive peak onset and offset seen in the average ERP. The figure also shows separate component ERP averages of the first and second halves of the trials for each subject, revealing a slightly reduced FP3 amplitude in the second as compared to the first half of trials $\left(t_{20}=1.73, p=0.098\right)$. A similar habituation effect was not observed for Group 2, where the average ERP amplitude was not significantly different between first and second half of all trials $\left(t_{18}=0.45, \mathrm{~ns}\right)$. The same analysis was also performed for the FP3 cluster in the rare tone responses, and for PP3 for the rare sounds and the rare 
FP3 cluster ERPs

Group 1: nontarget novel sounds Group 2: target novel sounds
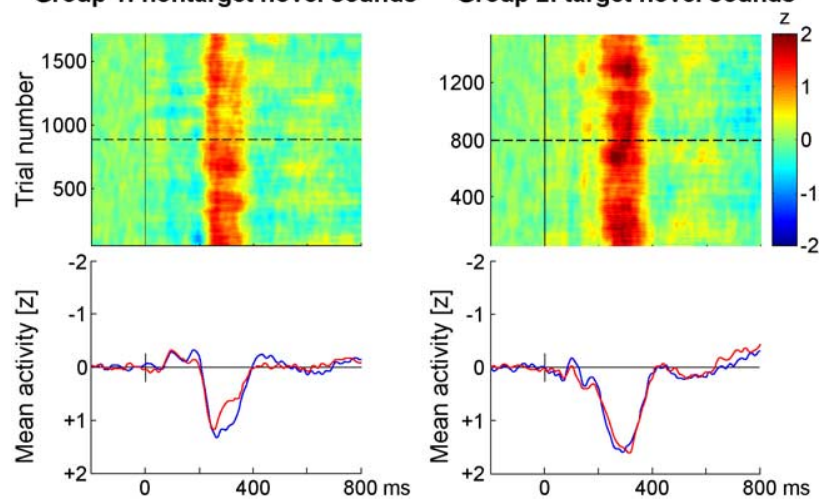

Fig. 4. ERP-image plots of FP3 component activity in single trials, showing trial-by-trial changes in ERP responses (habituation) to rare novel sounds, separately for Group 1 (left column) and Group 2 (right column). Top: normalized single trials in ERP-image plots are shown sorted by original trial order, and with a (vertical) smoothing moving window (100 trials). The color-coded amplitudes in the ERP-image plots show the low variability of FP3 component activity across all trials (1773 for Group 1, and 1591 for Group 2). Bottom: grand mean FP3 component activity average for the first (blue) and second (red) half of all trials (the average of all trials below and above the dashed horizontal lines in the ERP images, respectively). Data shown were $z$-normalized to unit baseline variance to facilitate comparison across components by dividing each trial by the standard deviation of the component activity in the -200 to 0 ms prestimulus interval.

tone responses (not shown). None of these analyses indicated habituation effects.

\section{Discussion}

The present study investigated the role of task relevance on the novelty P3 ERP. Commonly, task irrelevance has been considered a necessary condition for stimuli to elicit a novelty P3 ERP. The present study provides clear evidence against this view. Specifically, novelty P3 peaks were elicited by both task-relevant and task-irrelevant unique environmental sounds embedded in an auditory oddball series. Results of initial analysis of the scalp ERPs in this experiment paralleled recent research, showing that the novelty P3 is larger for taskrelevant than task-irrelevant environmental sounds [19]. However, spatiotemporal overlap of ERP components is a well-recognized problem [2], in particular with respect to novelty P3 and P3b ERP components $[39,40]$. ICA, applied to concatenated single-trial data of the auditory novelty oddball, revealed that the novelty P3 amplitude enhancement was at least partly due to an overlapping P3b-related process. However, before further conclusions on the functional significance of the novelty P3 can be drawn, the approach employed merits some discussion.

\subsection{Methodological issues}

Independent component analysis (ICA) is a relatively new linear decomposition technique that can be regarded as refining the goal of PCA-based approaches to separate the observed EEG signals into both physiologically and behaviorally distinct components [8]. ICA decomposes EEG data such that the resulting component activities have minimal mutual information. Independent components are often characterized by scalp maps fitting the projection of a single equivalent current dipole, which is compatible with the assumption that each independent component reflects synchronous local field activity of a connected patch of cortex. Mathematically, this is achieved by maximizing the joint entropy of independent components [4]. Note that the concept of statistical independence goes far beyond statistical orthogonality. It implies that two or more variables are not only uncorrelated (i.e., orthogonal), but also that all higher order moments are zero, or, practically speaking, close to zero. From a more practical point of view, another advantage is that ICA can be applied to low density EEG data as well. High-density recordings may be beneficial for ICA decompositions, but reasonable results can also be obtained with sparse montages [31].

ICA has been reported previously to successfully disentangle EEG and ERP components both when applied to averaged data [29] and to concatenated single-trial data $[7,23,31,33]$. In the present study, application of ICA disclosed a variety of interesting results which confirm and extend previous efforts to disentangle novelty P3 and P3b using PCA $[10,20,39,40]$. However, PCA is usually applied to averaged ERPs [2,3,9], whereas in the present study ICA was employed on concatenated single-trial data separately for each subject. Overall, the present findings strongly suggest that the main features of averaged ERP components novelty P3 and P3b can be successfully separated via ICA single-trial decomposition into independent EEG processes.

In comparison to PCA-derived ERP component measures (e.g., Ref. [2]), the amount of variance explained by FP3 and PP3 clusters may be considered relatively low. Note, however, that ICA tends to distribute data variance much more equally across all components than PCA [8]. Note also that the FP3 and PP3 components were separated from the whole concatenated single-trial EEG data, and thus may reflect not only the phase imbalances in ongoing EEG that sum to produce the average ERP [28]. We regard the FP3 and PP3 clusters as prototypical representations of EEG processes accounting for major portions of the novelty P3 and P3b ERP peaks. Note, however, that additional brain processes contributing to novelty $\mathrm{P} 3$ generation may not be captured by the FP3 cluster.

\subsection{Association between ICA components and ERP components}

Labeled ERP peaks (ERP 'components') are usually defined by the latency range over which they are expressed, their scalp topography, and differences in their response to 
changes in experimental variables [11]. The ERP contribution of the FP3 cluster reflected all the features of the novelty P3 ERP peak, which is elicited in response to contextually salient, normally task-irrelevant stimuli, and has a positive peak at about $300 \mathrm{~ms}$ with a fronto-central scalp distribution [18]. The ERP contribution of the PP3 cluster, on the other hand, expressed the main characteristics associated with the classical P300, which is elicited by rare, typically task-relevant stimuli and has a parietal deflection at latencies between 300 and $600 \mathrm{~ms}$ [12]. Furthermore, the two identified clusters explained a considerable amount of variance in the ERP data (one-third to one-half). The observation that both independent processes contributed to both experimental conditions contradicts classical assumptions, but agrees with other recent studies reporting similar results $[20,40]$.

Comparing the scalp novelty P3 ERP peak with the evoked response features expressed by the FP3 independent component cluster revealed some interesting aspects. First, the novelty P3 deflection in these data was characterized by a biphasic waveform, at least for Group 1. Escera et al. [13] reported that the novelty P3 is a complex consisting of at least two positive peaks, one early ( $230 \mathrm{~ms})$ and one somewhat later $(\sim 315 \mathrm{~ms})$, with the latter being larger when stimuli are covertly attended. The present findings for scalp ERPs parallel this observation. In our data, the novelty P3 ERP elicited by rare sounds clearly differed between groups at the latency of the second peak, with Group 2 showing the larger novelty P3. Correspondingly, the positive peak expressed in the FP3 independent component cluster average showed a latency difference between groups (260 versus $308 \mathrm{~ms}$ ). Note, however, that we did not find a significantly larger FP3 ERP activity in Group 2 than in Group 1, giving rise to the assumption that the spatiotemporally overlapping P3b-related process accounted at least partly for the group differences seen in the scalp ERPs.

In both groups, a clear negative peak preceding the main positive potential was evident in the FP3 component cluster ERP, which may contribute to the auditory N1 ERP component. This unexpected finding offers at least two interpretations: a region that produces synchronized temporal activity during and/or between trials will be modeled by the same independent component. In other words, it is possible that ICA did not successfully disentangle temporally distinct but spatially highly overlapping brain processes, perhaps because (disregarding polarity) auditory $\mathrm{N} 1$ scalp topography is very similar to the novelty P3 scalp topography. An alternative possibility is that the cortical region involved in novelty $\mathrm{P} 3$ generation could also generate a portion of the auditory N1 peak. (Note that Makeig et al. [33] identified nine independent classes of processes in frontal, parietal and occipital cortex contributing to the visual N1 ERP peak.) Direct evidence for the latter interpretation comes from a magnetoencephalographic study reporting that the magnetic novelty P3 is generated in the auditory cortex [1]. Furthermore, by combining ERPs and functional magnetic resonance imaging (fMRI), Opitz et al. [35] also identified bilateral superior temporal lobe areas as being involved in generating the novelty P3, which suggests that the auditory cortex, involved in N1 generation, could be part of a network processing stimulus novelty.

If one agrees with the assumption that the PP3 cluster contributes a prototypical portion of the P3b ERP component, then both target and nontarget deviant stimuli elicited a P3b. Accordingly, it seems that task relevance is not a necessary condition to elicit a P3b [12]. On the basis of spatiotemporal PCA-based decompositions of ERPs in the novelty oddball paradigm, the same conclusion has been made previously [20,40]. It therefore appears that this finding is replicable across different laboratories employing different decomposition approaches.

\subsection{Novelty P3 unity}

ICA did not identify two separate clusters reflecting the two novelty P3 peak features. This is particularly noteworthy with regard to the ongoing discussion about whether the P3a and novelty P3 are separate ERP features [18,37]. In Group 2, the latency of the P3a-like response to rare tones $(320 \mathrm{~ms})$ matched the latency of the (second peak of the) novelty P3 component elicited by the novel environmental sounds (320 $\mathrm{ms})$. Further inspection of the FP3 cluster ERP time course does not suggest a clear distinction between $\mathrm{P} 3 \mathrm{a}$ and novelty $\mathrm{P} 3$, which again is in good agreement with previous evidence $[37,40]$. However, future research dissociating the two peaks of the novelty P3 might profit from analyzing high-density electrode arrays and/or from limiting ICA training data solely to the novelty condition. This approach should help to determine whether P3a and novelty P3 are (spatially) identical components, as suggested by the present data. However, the FP3 ICA cluster likely reflects only one (major) aspect involved in novelty $\mathrm{P} 3$ generation. A more comprehensive ICA clustering approach [33] might help to better understand the temporal dynamics of novelty processing.

\subsection{Habituation}

Another interesting aspect of the FP3 cluster is its timeon-task behavior. It is well known that the novelty P3 amplitude habituates $[5,6,18]$, and this was partly reflected in the FP3 cluster. The findings for Group 1 may be regarded as a replication of previous habituation reports. Here, a tentative FP3 ERP response habituation effect was found for novel environmental sounds when they were nontargets. The relatively small size of this habituation effect (in comparison to our previous study, [6]) might be related to the presentation of a few tone and novel sound stimuli prior the recording of the first block. This may have elicited novelty P3 habituation processes [18] and reduced the chance to observe habituation over the course of the experiment. For Group 2, the novel environmental sounds 
were designated as targets, and interestingly, there was no indication of FP3 ERP habituation. Recently, Gaeta et al. [19] proposed that habituation of the novelty P3 is induced by the invariant target. This consideration is in good agreement with our findings, as invariant targets were presented to Group 1, and only this group showed FP3 habituation. To what extent task relevance reliably inhibits habituation of the novelty $\mathrm{P} 3$ remains to be determined.

\subsection{Dipole locations of independent components}

In the present report, dipole localization was used primarily as a measure of component quality. However, the single equivalent dipole locations for the independent components contributing to the FP3 cluster are consistent with the assumption that the anterior cingulate is part of a widely distributed network involved in novelty processing. This view is in good agreement with previous EEG studies aiming at localizing novelty P3 generators, using sparse $[34,42]$ and high-density montages [10]. The inconsistency with the above-reported Opitz et al. [35] finding may reflect that several cortical regions contribute to the novelty P3 ERP $[18,42]$, including superior temporal and anterior cingulate areas. In light of previous research, an FP3 equivalent dipole location close to the anterior cingulate seems reasonable, as there is no doubt that frontal cortex areas play an important role for novelty processing (e.g., Refs. [25,26]).

Dipole localization of the PP3 cluster was spatially less consistent than dipole localization of the FP3 cluster. Furthermore, as indicated by the PP3 cluster 2-D topography, these components may in some cases be better modeled by two symmetrical bilateral dipoles. However, Makeig et al. [33] also report a parietal cluster of independent components contributing to the visual P3b, whose equivalent current dipoles are spatially widely distributed. Recently, dipole modeling of the P300 was performed using two bilateral dipoles located close to the temporoparietal junction [10], presumably coupled via corpus callosum. Several brain imaging studies investigating the oddball paradigm reported bilateral activation of the temporoparietal junction for rare targets, among other activations [25,27], and bilateral activation of temporoparietal cortex may be the most consistent finding across brain imaging studies that employed an oddball paradigm [10]. However, since the cluster identified here accounted for a relatively small portion of the P3b peak, it seems premature to discuss the relation of the PP3 independent component cluster to the temporoparietal junction. In light of the present fMRI literature, however, the temporal-parietal junction is part of the complex network involved in P300 generation [10,18].

\subsection{Functional significance of the novelty P3}

What are the implications of the present findings regarding the functional significance of the novelty P3? In contrast to current theoretical models but in agreement with recent empirical work [19], the present study contradicts the view that task irrelevance is necessary to evoke a novelty P3 ERP.

Gaeta et al. [19] recently manipulated stimulus characteristics and task relevance in a modified version of the auditory novelty oddball paradigm by presenting two low probability stimulus series, unique complex environmental sounds and unique pure tones of varying frequency. For one subject group, rare unique environmental sounds were designated as targets, and rare unique tones served as nontarget deviants. For a second group, target and nontarget stimuli were reversed, that is, unique sounds now served as nontarget deviants and unique tones as targets. Environmental sounds serving as targets elicited a novelty P3 that was significantly larger than the novelty P3 elicited by nontarget deviant sounds. At first glance, this finding seems in clear contradiction with ASM and RIM, which both imply that task irrelevance is necessary to elicit a novelty P3. Indeed, based on this and other results (e.g., Ref. [21]), the question raised whether novelty processing, as reflected by the novelty P3 component, can be enhanced by voluntary attention. However, previous data $[5,19]$ clearly indicate that task-relevant novel stimuli also elicited a larger posterior $\mathrm{P} 3 \mathrm{~b}$ as compared to nontarget novel stimuli. Because novelty P3 and P3b overlap in space and time (e.g., Refs. $[39,40])$, it cannot be excluded that the enhanced novelty P3 for attended novel stimuli was at least partly due to an overlapping $\mathrm{P} 3 \mathrm{~b}$ in these studies.

By means of ICA decomposition, the present study now provides evidence that the larger amplitude of the novelty P3 ERP peak following target novel environmental sounds can partly be attributed to an overlapping P3b-related process. In addition, there was no indication of systematic differences between FP3 clusters identified for Group 1 and Group 2. Although we are aware of the difficulties inherent in the interpretation of a null hypothesis, we assume that novelty processing is not suppressed by voluntary attention. This is incompatible with the ASM model of Escera et al. [13] which states that the novelty $\mathrm{P} 3$ reflects attention switching from an attended to an unattended, task-irrelevant event. It is also incompatible with the RIM of Goldstein et al. [20] suggesting that the novelty $\mathrm{P} 3$ reflects a manifestation of a response inhibition process partially activated by a deviance detection mechanism. Group 2 in the present study was neither required to switch attention nor to inhibit a response. Nevertheless, they showed a novelty P3 to unique, dissimilar, and in this sense novel environmental sounds. Therefore, we suggest that it is stimulus novelty rather than the lack of expectancy that is reflected in the novelty $\mathrm{P} 3$.

The assumption that the novelty $\mathrm{P} 3$ reflects an automatic process, which is also inherent to the accounts by Escera et al. [14] and Goldstein et al. [20], is in good agreement with the current study. Some further considerations may help to design future work on novelty processing. First, the attention switching view of Escera et al. is based on studies in which subjects voluntarily attend to stimuli in the visual sensory modality while distractor stimuli eliciting a novelty 
P3 are presented in the auditory modality [15]. This points to the important question as to whether the mechanisms of attention switching between sensory modalities are the same as those responsible for attention switching within one modality. Additionally, it will be necessary to specify whether the novelty P3 represents manual response-related activity, as suggested recently [10]. Given participants were counting, the present study could not address the idea that the novelty P3 is related to manual response inhibition. However, this could be easily addressed in future studies by systematic comparisons of stimulus- and response-locked brain-electrical activity to novel environmental sounds designated as targets and nontargets.

In sum, the current study provides evidence that ICA, applied to concatenated single-trial EEG data, successfully decomposes the spatiotemporally overlapping ERPs novelty $\mathrm{P} 3$ and $\mathrm{P} 3 \mathrm{~b}$ into a range of underlying EEG processes. As revealed by analysis of the ICA-derived respective prototypical independent components, FP3 and PP3, task irrelevance is not an antecedent condition for eliciting a novelty $\mathrm{P} 3$. Rather, it seems that the novelty $\mathrm{P} 3$ peak reflects an automatic brain process related to the detection of stimulus novelty or salience.

\section{Acknowledgments}

The present study was planned and carried out at the Institute of Medicine, Research Center Juelich, Germany, whose support is gratefully acknowledged. We thank O. Haumann and C. Kranczioch for assistance in data recording, and A. Schirmer for helpful comments on an earlier version of the manuscript. This report was supported by The Swartz Foundation through a visiting fellowship (S.D.) at the Swartz Center for Computational Neuroscience, University of California San Diego, La Jolla, CA.

\section{References}

[1] K. Alho, I. Winkler, C. Escera, M. Huotilainen, J. Virtanen, I.P. Jääskeläinen, E. Pekkonen, R.J. Ilmoniemi, Processing of novel sounds and frequency changes in the human auditory cortex: magnetoencephalographic recordings, Psychophysiology 35 (1998) $211-224$.

[2] A. Beauducel, S. Debener, Misallocation of variance in event-related potentials: simulation studies on the effects of test power, topography, and baseline-to-peak versus principal component quantifications, J. Neurosci. Methods 124 (2003) 103-112.

[3] A. Beauducel, S. Debener, B. Brocke, J. Kayser, On the reliability of augmenting/reducing: peak amplitudes and principal components analysis of auditory evoked potentials, J. Psychophysiol. 14 (2000) $226-240$.

[4] A.J. Bell, T.J. Sejnowski, An information-maximization approach to blind separation and blind deconvolution, Neural Comput. 7 (1995) $1129-1159$

[5] E. Courchesne, S.A. Hillyard, R. Galambos, Stimulus novelty, task relevance, and the visual evoked potential in man, Electroencephalogr. Clin. Neurophysiol. 39 (1975) 131-141.
[6] S. Debener, C. Kranczioch, C.S. Herrmann, A.K. Engel, Auditory novelty oddball allows reliable distinction of top-down and bottom-up processes of attention, Int. J. Psychophysiol. 46 (2002) 77-84.

[7] A. Delorme, S. Makeig, EEG changes accompanying learned regulation of 12-Hz EEG activity, IEEE Trans. Neural Syst. Rehabil. Eng. 11 (2003) 133-137.

[8] A. Delorme, S. Makeig, EEGLAB: an open source toolbox for analysis of single-trial EEG dynamics including independent component analysis, J. Neurosci. Methods 134 (2004) 9-21.

[9] J. Dien, Addressing misallocation of variance in principal components analysis of event-related potentials, Brain Topogr. 11 (1998) $43-55$.

[10] J. Dien, K.M. Spencer, E. Donchin, Localization of the event-related potential novelty response as defined by principal components analysis, Cogn. Brain Res. 17 (2003) 550-637.

[11] E. Donchin, W. Ritter, W.C. McCallum, Cognitive psychophysiology: the endogenous components of the ERP, in: E. Callaway, P. Tueting, S.H. Koslow (Eds.), Brain event-related potentials in man, Academic Press, New York, 1978, pp. 349-441.

[12] E. Donchin, K.M. Spencer, J. Dien, The varieties of deviant experience: ERP manifestations of deviance processors, in: G.J.M. van Boxtel, K.B.E. Bocker (Eds.), Brain and Behaviour: Past, Present, and Future, University Press, Tilburg, The Netherlands, 1997, pp. 67-91.

[13] C. Escera, K. Alho, I. Winkler, R. Naatanen, Neural mechanisms of involuntary attention to acoustic novelty and change, J. Cogn. Neurosci. 10 (1998) 590-604.

[14] C. Escera, K. Alho, E. Schroger, I. Winkler, Involuntary attention and distractibility as evaluated with event-related brain potentials, Audiol. Neuro-otol. 5 (2000) 151.

[15] C. Escera, E. Yago, K. Alho, Electrical responses reveal the temporal dynamics of brain events during involuntary attention switching, Eur. J. Neurosci. 14 (2001) 877-883.

[16] M. Fabiani, V.A. Kazmerski, Y.M. Cycowicz, D. Friedman, Naming norms for brief environmental sounds: effects of age and dementia, Psychophysiology 33 (1996) 462-475.

[17] T.C. Ferree, P. Luu, G.S. Russell, D.M. Tucker, Scalp electrode impedance, infection risk, and EEG data quality, Clin. Neurophysiol. 112 (2001) 536-544.

[18] D. Friedman, Y.M. Cycowicz, H. Gatea, The novelty P3: an eventrelated brain potential (ERP) sign of the brain's evaluation of novelty, Neurosci. Biobehav. Rev. 25 (2001) 355-373.

[19] H. Gaeta, D. Friedman, G. Hunt, Stimulus characteristics and task category dissociate the anterior and posterior aspects of the novelty P3, Psychophysiology 40 (2003) 198-208.

[20] A. Goldstein, K.M. Spencer, E. Donchin, The influence of stimulus deviance and novelty on the P300 and novelty P3, Psychophysiology 39 (2002) 781-790.

[21] J.S. Holdstock, M.D. Rugg, The effect of attention on the P300 deflection elicited by novel sounds, J. Psychophysiol. 9 (1995) 18-31.

[22] T.P. Jung, S. Makeig, C. Humphries, T.W. Lee, M.J. McKeown, V. Iragui, T.J. Sejnowski, Removing electroencephalographic artifacts by blind source separation, Psychophysiology 37 (2000) 163-178.

[23] T.P. Jung, S. Makeig, M. Westerfield, J. Townsend, E. Courchesne, T.J. Sejnowski, Analysis and visualization of single-trial event-related potentials, Hum. Brain Mapp. 14 (2001) 166-185.

[24] V.A. Kazmerski, D. Friedman, Repetition of novel stimuli in an ERP oddball paradigm: aging effects, J. Psychophysiol. 9 (1995) $298-311$.

[25] K.A. Kiehl, K.R. Laurens, T.L. Duty, B.B. Forster, P.F. Liddle, Neural sources involved in auditory target detection and novelty processing: an event-related fMRI study, Psychophysiology 38 (2001) 133-142.

[26] R.T. Knight, Decreased response to novel stimuli after prefrontal lesions in man, Electroencephalogr. Clin. Neurophysiol. 59 (1984) $9-20$.

[27] D.E. Linden, D. Prvulovic, E. Formisano, M. Vollinger, F.E. Zanella, R. Goebel, T. Dierks, The functional neuroanatomy of target 
detection: an fMRI study of visual and auditory oddball tasks, Cereb. Cortex 9 (1999) 815-823.

[28] S. Makeig, A.J. Bell, T.P. Jung, T.J. Sejnowski, Independent component analysis of electroencephalographic data, in: D. Touretzky, M. Mozer, M. Hasselmo (Eds.), Advances in Neural Information Processing Systems, vol. 8, MIT Press, Cambridge, 1996, pp. $145-151$.

[29] S. Makeig, T.P. Jung, A.J. Bell, D. Ghahremani, T.J. Sejnowski, Blind separation of auditory event-related brain responses into independent components, Proc. Natl. Acad. Sci. U. S. A. 94 (1997) 10979-10984.

[30] S. Makeig, M. Westerfield, T.P. Jung, J. Covington, J. Townsend, T.J. Sejnowski, E. Courchesne, Functionally independent components of the late positive event-related potential during visual spatial attention, J. Neurosci. 19 (1999) 2665-2680.

[31] S. Makeig, M. Westerfield, T.P. Jung, S. Enghoff, J. Townsend, E. Courchesne, T.J. Sejnowski, Dynamic brain sources of visual evoked responses, Science 295 (2002) 690-694.

[32] S. Makeig, S. Debener, J. Onton, A. Delorme, Mining event-related brain dynamics, Trends Cogn. Sci. 8 (2004) 204-210.

[33] S. Makeig, A. Delorme, M. Westerfield, T.P. Jung, J. Townsend, E. Courchesne, T.J. Sejnowski, Electroencephalographic brain dynamics following manually responded visual targets, Public Libr. Sci. Biol. 2 (2004) 747-762.
[34] A. Mecklinger, P. Ullsperger, The P300 to novel and target events: a spatiotemporal dipole model analysis, NeuroReport 7 (1995) $241-245$.

[35] B. Opitz, A. Mecklinger, A.D. Frederici, D.Y. von Cramon, The functional neuroanatomy of novelty processing: integrating ERP and fMRI results, Cereb. Cortex 9 (1999) 379-391.

[36] C. Ranganath, G. Rainer, Neural mechanisms for detecting and remembering novel events, Nat. Rev., Neurosci. 4 (2003) 193-202.

[37] R.F. Simons, F.K. Graham, M.A. Miles, X. Chen, On the relationship of P3a and the novelty P3, Biol. Psychol. 56 (2001) 207-218.

[38] E.N. Sokolov, Perception and the Conditioned Reflex, Pergamon, Oxford, 1963.

[39] K.M. Spencer, J. Dien, E. Donchin, A componential analysis of the ERP elicited by novel events using a dense electrode array, Psychophysiology 36 (1999) 409-414.

[40] K.M. Spencer, J. Dien, E. Donchin, Spatiotemporal analysis of the late ERP responses to deviant stimuli, Psychophysiology 38 (2001) $343-358$.

[41] N.K. Squires, K.C. Squires, S.A. Hillyard, Two varieties of longlatency positive waves evoked by unpredictable auditory stimuli in man, Electroencephalogr. Clin. Neurophysiol. 38 (1975) 387-401.

[42] E. Yago, C. Escera, K. Alho, M.H. Giard, J.M. Serra-Grabulosa, Spatiotemporal dynamics of the auditory novelty P3 event-related brain potential, Cogn. Brain Res. 16 (2003) 383-390. 